\title{
Parallel scanning of physical and category information
}

\author{
DAVID BURROWS \\ State University of New York, College at Brockport, Brockport, New York 14420 \\ and \\ RONALD OKADA \\ Atkinson College, York University, Toronto, Canada
}

\begin{abstract}
In Sternberg's memory scan paradigm, subjects were required to scan for category membership or physical identity. In Experiment I, the scan rate when checking for either type of relationship at the same time was less than the sum of the scan rates when checking for either relationship separately. In Experiment II, presenting memory sets consisting of items from a single category affected the category scan function but not the physical scan function. The results were interpreted as evidence for the capacity to conduct parallel scans of independent memory encodings when retrieving remembered information.
\end{abstract}

This paper is concerned with the processes involved when people retrieve information from memory. In particular, it is concerned with whether people can retrieve several different pieces of information from memory simultaneously by coding these pieces of information into independent memory codes.

Sternberg $(1966,1969)$ argued that retrieval from short-term memory consists of a serial search process in which memory items are checked one at a time. This argument is based on experiments in which a subject is required to decide whether or not a test item was present in a short, memorized list. Reaction time (RT) of the two-choice decision is usually a linearly increasing function of the number of items in the memorized list. The linearity suggests a serial search in which the test item is compared with each memory item. Each additional comparison requires an additional unit of time, and the slope of the function provides an estimate of the average time per comparison. Burrows and Okada (1973) suggested that subjects might be able to perform at least two such serial scans simultaneously, provided that the scans are of two distinct memory codes. Subjects in three different conditions were required to decide if a test item was the same item as any memory set item, a synonym of any memory set item, or either the same as or a synonym of any memory item. Subjects in the same or synonym condition were required to make two sets of comparisons, one involving formal or physical characteristics of the memory items and the other involving semantic or meaning characteristics of the memory items. The average time required to make one formal comparison and one semantic comparison was estimated by the slope of the RT vs. memory set size function. The average time for a formal comparison

Requests for reprints should be sent to David Burrows, Department of Psychology, State University of New York, College at Brockport, Brockport, New York 14420. Preparation of this report was facilitated by Grant A8266 from the National Research Council of Canada to the second author. was measured by the slope for the same condition and the time for a semantic comparison was measured by the slope for the synonym condition. The slope for the same or synonym condition was equal to the average of the slopes for the other two conditions, suggesting a parallel scan process in which formal and semantic comparisons overlap in time. If subjects were not able to scan simultaneously for formal and semantic information but first had to do one comparison and then the other, the slope for the same or synonym condition would have been equal to the sum of the other two slopes. This would always be true for negative responses and true for positive responses if scanning were completely exhaustive. Parallel scanning can form the basis of a powerful retrieval scheme but, before general retrieval models can be constructed, the generality of the phenomenon must be established. In the Burrows and Okada (1973) experiment, the two sets of items being scanned were formal representations of words and semantic representations of the same words. These two representations (words and their meanings) are closely related, and it is possible that parallel scanning cannot be accomplished when the representations being scanned are truly independent. Experiment I attempted to demonstrate parallel scanning for formal features of memory items (physical identity) and semantic features of categories (category membership). This would show that the phenomenon is not limited to codes as closely related as words and their meanings. Experiment II attempted to demonstrate the independence of the codes being scanned in parallel by showing that the category retrieval component of a parallel scan can be altered without affecting the physical retrieval component.

\section{EXPERIMENT I}

In Experiment I, subjects in three groups had to classify each test item as: an exact match of any 
memory item, a category exemplar of any memory item, or either an exact match or a category exemplar.

\section{Method}

Subjects. The subjects were 24 students, both men and women, enrolled in a general psychology course at the State University College at Brockport. Participation in the experiment was rewarded by adding one percentage point to each student's course grade.

Apparatus. Stimuli were recorded in a male voice on a Wollensak tape recorder and presented by Wollensak headphones. When test items were presented, prerecorded signals were read from the magnetic tape by a Uher diapilot, Model F422. The diapilot closed a relay which started a Standard electric timer. The subject signaled his response and stopped the timer by pressing one of two pushbuttons. The pushbuttons were mounted in a horizontal orientation in a box placed on the armrest of the subject's chair. The timer measured RTs to the nearest $.01 \mathrm{sec}$. Depressing either pushbutton also turned on one of two neon lamps which indicated to the experimenter which response the subject had made.

Procedure. Each trial began with the presentation of a memory list of one, two, three, or four words, presented sequentially at a rate of three words per $2 \mathrm{sec}$. Memory set presentation was followed by a pause of $.667 \mathrm{sec}$ and then a test item. The subject had to classify the test item as a "yes" or a "no" item and push the appropriately labeled button. Each subject worked through four blocks of four practice trials and 24 test trials each, with memory list length fixed within each block. Twelve of the 24 test trials in a block required "yes" responses. Serial position of positive test items was balanced among all possible positions. The order in which the four blocks of fixed memory list length were presented was randomized across subjects. The subjects were divided into three groups. In the physical match group, the memory set items on each trial were sampled without replacement from a set of 20 category labels used in the Battig and Montague (1969) norms. The test item was also a category label, and subjects were instructed to respond "yes" if the test item was an exact match of any memory item and "no" otherwise. . On one-half of the trials for any memory list length, the test item was a category label presented in the memory set and on the remaining trials the test item was one of the category labels not presented in the memory set.

In the category match condition, the memory set consisted of one, two, three, or four category labels from the pool of 20 . The test item had to be classified as either a member of one of the categories in the memory set or not a member of any memory set category. On one-half of the trials for any memory set size, the test item was randomly sampled from the set of the five most frequent exemplars of one of the memory categories. On the remaining trials, the test item was an exemplar of one of the categories not presented in the memory set.

In the physical or category match condition, the memory set was composed of category labels from the set of 20 . Subjects were to respond "yes" to any test item that was either an exact match or a category exemplar of any memory item. On one-quarter of the trials, the test item was one of the category labels presented in the memory set; on one-quarter it was an exemplar of a memory set category; on one-quarter it was a category not presented in the set; and on the last one-quarter it was an exemplar of a category not presented in the memory set.

Two complete sets of trials for each of the three conditions were generated and randomly ordered. Four of the eight subjects in each condition worked through one set, and the remaining four worked through the other set. All subjects were familiarized with the list of categories used before testing began.

\section{Results}

Table 1 shows error proportions as a function of set size for the three conditions. The error proportions were subjected to an analysis of variance after being transformed with an arcsin transformation (Winer, 1962). None of the results from the analysis of variance approached significance. All error trials were discarded from the RT analysis.

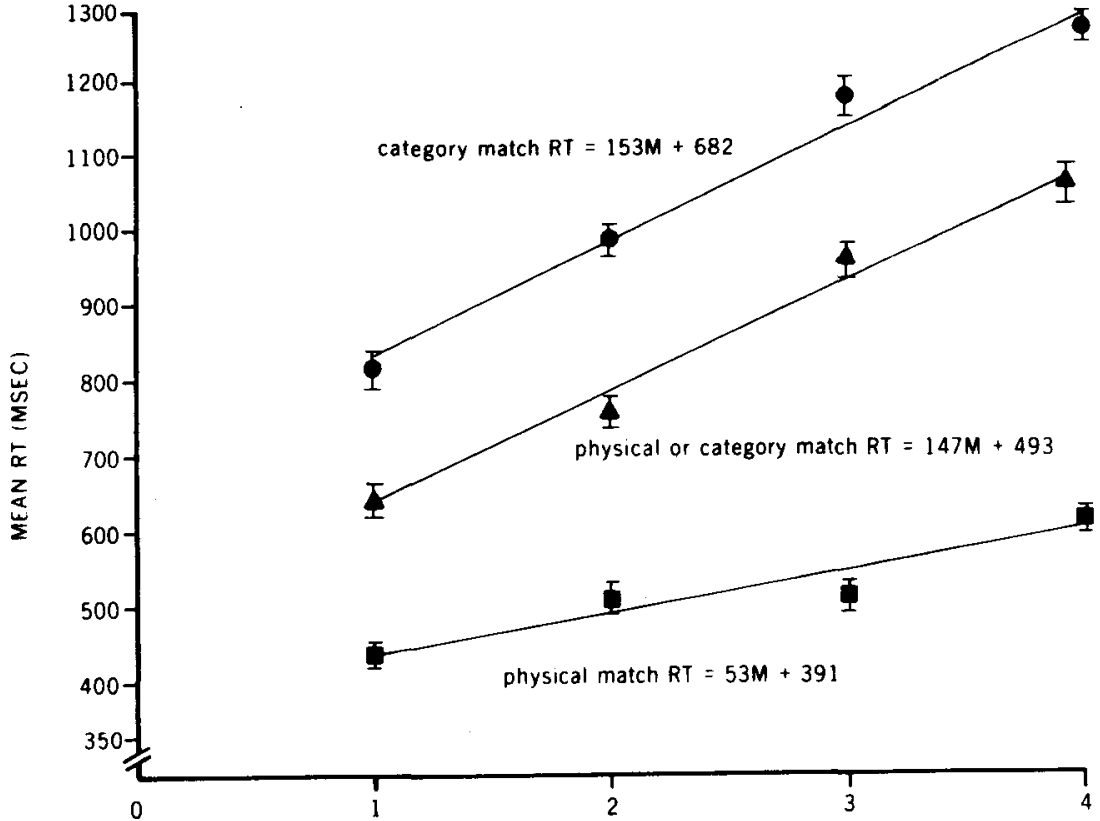

Figure 1. Mean reaction time as a function of memory list length for physical match, category match, and physical or category match conditions of Experiment l. Fitted lines and equations obtained by least-squares linear regression. The vertical bars indicate the values of the standard errors of mean correct RTs. 
Figure 1 shows mean RT as a function of set size for each of the three conditions. The straight lines were fitted by least-squares linear regression. The functions appear to be linear. This supports the hypothesis of retrieval by a serial scan in all conditions. The slopes for "yes" trials and "no" trials did not differ for either the physical match condition $[\mathrm{F}(1 / 4)=3.6$, NS] or the category match condition $[\mathrm{F}(1 / 4)=2.1$, NS $]$. For comparison purposes, the three types of trials in the physical or category match condition were combined, as shown in Figure 1. A breakdown of the physical or category match condition is presented below.

Inspection of Figure 1 shows that the slope of the physical or category match condition, $147 \mathrm{msec} / \mathrm{item}$, is less steep than the sum of the slopes for the other two conditions, $206 \mathrm{msec} /$ item. This difference was statistically reliable by a $t$-test (Draper \& Smith, 1966), $[\mathrm{t}(2)=4.8, \mathrm{p}<.025]$. This supports the hypothesis that subjects in the physical or category match condition are able to execute the two scans in an overlapping fashion. For the two simple conditions, the category match condition had a steeper slope than the physical match condition, $153 \mathrm{msec} /$ item vs. $53 \mathrm{msec} / \mathrm{item}$. The finding of a slower scanning rate for semantic information than for formal information is consistent with data from Burrows and Okada (1973) and Juola and Atkinson (1971).

Within the physical or category match condition there are three types of trials: physical match, category match, or no match. The RT vs. set size slope for physical match trials within the physical or category condition was $71 \mathrm{msec} / \mathrm{item}$, which is remarkably close to the value of $70 \mathrm{msec} /$ item for positive trials in the physical match condition. The two slopes were not significantly different, $F<1$. The slope for the category match trials within the physical or category match condition was $162 \mathrm{msec} /$ item. This was not different from the slope of $133 \mathrm{msec} /$ item for positive trials in the category condition $[F(1 / 4)=1.5, N S]$. Finally, the slope for negative responses within the physical or category condition was $185 \mathrm{msec} / \mathrm{item}$, which was not different from the slope of $169 \mathrm{msec} / \mathrm{item}$ for negative responses in the category condition, $\mathrm{F}<1$.

The equivalence between the slopes of physical and category match trials in the physical or category condition and the slopes for the corresponding trial types in the simple conditions is further evidence for parallel scanning. The equivalence suggests that processing of one type of information is not slowed by the simultaneous processing of the other type of information.

\section{Discussion}

The results indicate that subjects in the physical or category match condition are able to take advantage of alternative memory codes to improve the efficiency of memory retrieval. In the physical or category match
Table 1

Error Proportions in Experiment I as a Function of Memory List Length for the Physical Match, Category Match, and Physical or Category Match Conditions

\begin{tabular}{lcccc}
\hline & \multicolumn{5}{c}{ Memory List } & \multicolumn{3}{c}{ Length } \\
Condition & 1 & 2 & 3 & 4 \\
\hline Physical Match & .04 & .02 & .03 & .05 \\
Category Match & .07 & .03 & .05 & .06 \\
Physical or Category Match & .07 & .09 & .07 & .09 \\
\hline
\end{tabular}

condition, a subject must attempt both a formal and a semantic comparison between the test item and each memory item. The slope values suggest that both comparisons can be completed in $147 \mathrm{msec}$. Done separately, a formal comparison requires $53 \mathrm{msec} / \mathrm{item}$ and a semantic or category comparison requires $153 \mathrm{msec} / \mathrm{item}$. Making both comparisons in series would require $206 \mathrm{msec} / \mathrm{item}$. It is clear that the two comparisons must be overlapping in time, and that parallel scanning of formal and semantic information is possible.

The breakdown of the data for the physical or category match condition suggests that parallel scanning proceeds in the following way: the subject simultaneously initiates a physical and a category scan when presented with the test item. If the test item is physically identical to any memory item, a physical match is detected and a positive response is initiated as soon as all physical comparisons are completed. This results in the equivalence between the physical match slope in the complex condition and the slope in the simple physical match condition. If the test item is categorically identical to any memory item, a category match is detected and a positive response is initiated as soon as all category comparisons are completed. This results in the equivalence between the category match slope in the complex condition and the corresponding slope in the simple category match condition. If the test item does not match any memory item, both the physical and category scans are completed without a match, and a negative response is initiated. The scan rate for negative decisions will be determined by the slower of the two component scans, namely the category scan. This results in the equivalence between the "no" slope in the physical or category condition and the "no" slope in the category condition.

\section{EXPERIMENT II}

Experiment II attempted to measure the extent to which two parallel scanning processes can operate on separate memory codes independently. The results of Experiment I do not rule out the possibility that subjects are carrying out two operations on a single code. Subjects may simply remember what words were in the list, making physical matches by direct comparisons between memory words and test item, and 
making category matches by comparisons between memory words and a categorized version of the test item.

Subjects could make more efficient use of parallel scanning if each operation is carried out on a separate memory code. Each memory code could be organized in a way that makes scanning of that particular code most efficient. For instance, if a memory list consists of four items belonging to a single category, a category code could be set up with a single entry, while the physical code maintains four entries. This is not possible if subjects use a single four-entry code for making both physical and category match decisions.

Experiment II attempted to show that presented information can be encoded into two unique codes that may be manipulated independently and scanned independently. Subjects were required to decide whether a test item was either an exact match of a memory item or belonged to the same category as any memory item. All items were exemplars of the 20 categories used in Experiment I. On "whole" trials, each item in the memory set belonged to a single category. Logically, this means that the number of category units does not increase with memory set size, while the number of physical items does. If category and physical codes can be searched independently, then RTs for category matches should be unaffected by memory set size, while RTs for physical matches should be affected. If category information cannot be manipulated independently, then category matches should increase with set size, as in Experiment I. Mixed in with the whole trials are "fragmented" trials on which each memory set item belonged to a different category. These were to provide a baseline for evaluating the effects of whole trials.

\section{Method}

Subjects. The subjects were 16 students selected from the same population as in Experiment $I$.

Apparatus. The apparatus was the same as used in Experiment 1 .

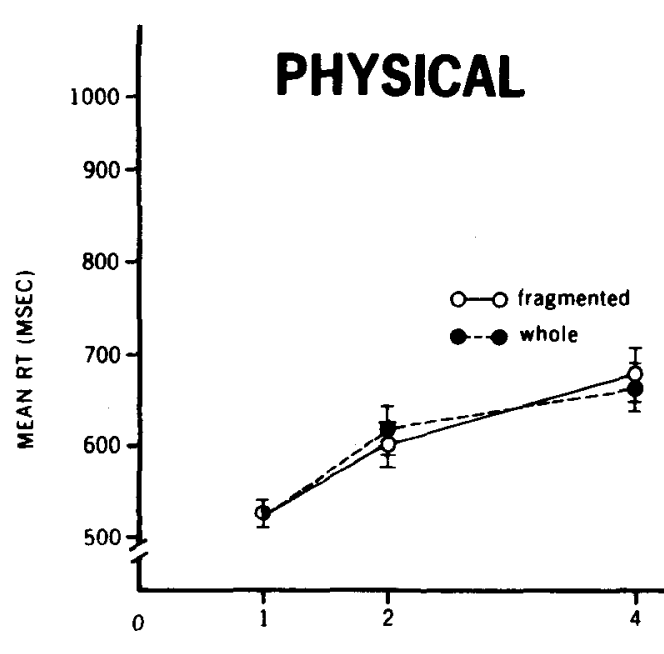

MEMORY SET SIZE
Procedure. Each trial began with the presentation of one, two, or four memory words, at a rate of three words per $2 \mathrm{sec}$. Following the memory set there was a pause and then a test item. Subjects were to respond positively if the test word was an exact match of any word in the memory set or a member of the same category as any memory set word. Each subject worked through three blocks of 24 trials with the number of memory words fixed throughout a block. Within a block there were eight trials on which the test word was an exact match of a memory word, eight on which it belonged to the same category as at least one of the memory set words, and eight on which it bore neither of the above relationships to any memory word. For two- and four-item blocks, one-half of the trials were "fragmented," with each memory item drawn from a different category, and one-half were "whole," with each item belonging to the same category. The order of whole and fragmented trials was random. All items were drawn from the pool of five most frequent exemplars of the 20 categories used. Two complete sets of materials were generated and recorded, with eight of the 16 subjects working through each set. The order of the three blocks of trials was randomized for each subject.

\section{Results}

Table 2 shows the error proportions for each set size and trial type combination. For completeness, Set Size 1 data are listed under both the fragmented and whole headings. The error rates for physical matches are low in all conditions. The error rates are also low for the category and "no" trials with whole presentation but are rather high in the fragmented condition.

The error data were converted with an arcsin transformation and analyzed as a three factorial design with factors of presentation type (whole vs. fragmented), memory list length, and match type (physical, category, or negative). Significant main effects of presentation type $[F(1 / 15)=9.4, p<.01]$, memory list length $[F(2 / 30)=19.8, p<.01]$, and match type $[F(2 / 30)=8.9, p<.01]$, were obtained. Also significant were the two-way interactions of Presentation Type by Memory List Length $[\mathrm{F}(2 / 30)=5.4, \mathrm{p}<.01]$, Presentation Type by Match Type $[\mathrm{F}(2 / 30)=3.7$, $\mathrm{p}<.05]$, and the triple interaction $[\mathrm{F}(4 / 60)=4.9$, $\mathrm{p}<.01]$.

\section{CATEGORY}

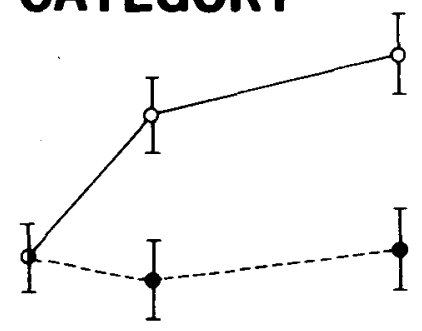

- - tragmented

- whole

Figure 2. Mean reaction time as a function of memory list length for whole and fragmented memory sets. Left panel for physical match trials and right panel for category match trials. The vertical bars indicate the values of the standard errors of mean correct RTs. 
Figure 2 shows mean correct RT as a function of set size for the two trial types of interest, category and physical matches, for each of the memory set types. The RTs for the physical matches rise monotonically with set size and are not affected by type of memory set. The best straight line functions have slopes of $39 \mathrm{msec} / \mathrm{item}$ and $44 \mathrm{msec} / \mathrm{item}$ for whole and fragmented trials, respectively.

The category match RTs are also increasing with set size for the fragmented memory sets with a slope of $70 \mathrm{msec} / \mathrm{item}$, but for whole memory sets show a decrease from Set Size 1 to 2 and a small increase from 2 to 4 . The best fitting straight line had a slope of $7 \mathrm{msec} /$ item. This slope was not significantly different from zero, $F<1$. Type of trial does affect category matches. Since the set size effects appear to depart from linearity, the data for the whole sets were analyzed by analysis of variance on the mean RTs for each subject, rather than comparing slopes. The analy sis demonstrated the differential effect of match type as a significant Match Type by Memory List Length interaction $[F(2 / 30)=5.26, p<.05]$.

Also significant were the main effects of match type $[F(1 / 15)=7.11, p<.05]$ and memory set size $[F(2 / 30)=22.9, p<.01]$. A separate analysis was performed on the negative trials for the fragmented and the whole presentation conditions. With fragmented presentation, RT increased with memory list length at a rate of $97 \mathrm{msec} /$ item. Memory list length did not affect the RT for negative responses with whole presentation; the best fitting straight line had a slope of $-0.7 \mathrm{msec} /$ item.

\section{DISCUSSION}

The results show that retrieval of category information from a memory set can be manipulated without affecting the retrieval of physical information from the same set. For all trials, the use of lists with all members belonging to a single category clearly reduced the time necessary for extracting category information without affecting the time necessary for extracting physical information. This implies the existence of separate memory codes underlying the two tasks. The assertion that subjects are able to create an efficient category code in the whole presentation condition is strengthened by the data on negative trials alone. When all memory items belong to a single category, the number of memory items does not affect mean RT, suggesting that subjects have set up a single item category code and can immediately initiate a negative response if the test item's category does not match this code.

In general, the error data effects can be interpreted as evidence that conditions which require more processing
Table 2

Error Proportions in Experiment Il as a Function of Memory List Length, With Separate Entries for Each Trial Type and Memory Set Type Combination

\begin{tabular}{|c|c|c|c|c|c|c|}
\hline \multirow[b]{4}{*}{ Test Type } & \multicolumn{6}{|c|}{ Trial Type } \\
\hline & \multicolumn{3}{|c|}{ Fragmented } & \multicolumn{3}{|c|}{ Whole } \\
\hline & \multicolumn{3}{|c|}{ List Length } & \multicolumn{3}{|c|}{ List Length } \\
\hline & 1 & 2 & 4 & 1 & 2 & 4 \\
\hline Physical Match & .02 & .03 & .00 & .02 & .03 & .05 \\
\hline Category Match & .12 & .20 & .16 & .12 & .03 & .05 \\
\hline No & .09 & .06 & .17 & .09 & .03 & .03 \\
\hline
\end{tabular}

and lead to longer RTs also lead to greater proportions of errors. The prediction that whole presentation should make category but not physical matches easier than fragmented presentation and that the superiority of whole presentation increases with the number of memory items implies that all three factors in the experiment should interact, as the analyses indicate they do.

The results of the two experiments are evidence for a powerful retrieval capacity; people can retrieve information from two codes at the same time and can manipulate one of these codes to make retrieval more efficient without affecting the other code. Such a mechanism may help to account for the apparent efficiency of memory retrieval in natural situations where the "memory set" is presumably quite large. It is possible that people encode information about an event in a number of ways. One encoding can reduce the information to a small set (as in categorization) while other encodings can preserve all of the specific details about the information. For many purposes, the information in the reduced or reorganized encoding may be sufficient. If all encodings are searched in parallel, the reduced encoding will yield an answer quite rapidly. Parallel scanning with independent codes may indeed be an important part of efficient information retrieval.

\section{REFERENCES}

Battig, W, F., \& Montague. W. E. Category norms for verbal items in 56 categories: $A$ replication and extension of the Connecticut category norms. Joumal of Experimental Psychology Monograph, 1969, 80, (3, part 2), 1-46.

Burrows, D., \& Okada, R. Parallel scanning of semantic and formal information. Journal of Experimental Psychology, $1973,97,254-257$.

Draper, N. R., \& Smith, H. Applied regression analysic. New York: Wiley, 1966.

Juola, J. F., \& Atkinson, R. C. Memory scanning for words versus categories: Journal of Verbal Learning and Verbal Behavior, 1971, 10, 522-527.

Sternberg, S. High-speed scanning in human memory. Science, $1966,153,652-654$.

Sternberg, S, Memory-scanning: Mental processes revealed by reaction-time experiments. American Scientist, 1969, 57, 421-457.

Winer. B. J. Statistical principles in experimental design. New York: McGraw-Hill, 1962.

(Received for publication December 5,1974 , Revision received April 2, 1975.) 